\title{
Implementation of Extended Kalman Filter for Real-Time Noncontact ECG Signal Acquisition in Android-Based Mobile Monitoring System
}

\author{
Vega Pradana Rachim ${ }^{1}$, Sung-Chul Kang ${ }^{2}$, Wan-Young Chung ${ }^{1}$, and Tae-Ha Kwon ${ }^{1,+}$
}

\begin{abstract}
Noncontact electrocardiogram (ECG) measurement using capacitive-coupled technique is a very reliable long-term noninvasive health-care remote monitoring system. It can be used continuously without interrupting the daily activities of the user and is one of the most promising developments in health-care technology. However, ECG signal is a very small electric signal. A robust system is needed to separate the clean ECG signal from noise in the measurement environment. Noise may come from many sources around the system, for example, bad contact between the sensor and body, common-mode electrical noise, movement artifacts, and triboelectric effect. Thus, in this paper, the extended Kalman filter (EKF) is applied to denoise a real-time ECG signal in capacitive-coupled sensors. The ECG signal becomes highly stable and noise-free by combining the common analog signal processing and the digital EKF in the processing step. Furthermore, to achieve ubiquitous monitoring, android-based application is developed to process the heart rate in a realtime ECG measurement.
\end{abstract}

Keywords: Noncontact ECG, Capacitive-coupled electrodes, Real-time EKF, Android application

\section{INTRODUCTION}

Electrocardiogram (ECG) signal, the small electrical signal produced by heart activity, is one of the major monitoring parameters of modern health care. This signal is one of the most representative health signals used to diagnose the patient conditions, such as measuring the rate and regularity of heartbeats and detecting the presence of any heart damage. Medical diagnosis using ECG signal can provide very important information about the heart status of the patients. Thus, the current trend is to develop this remote healthcare technology to provide healthy life anywhere and anytime. A promising technique is available to obtain ECG signal in daily life, which is called the capacitive-coupled ECG measurement system.

The capacitive-coupled ECG system, as one of the promising techniques for measuring electrocardiogram in remote areas,

${ }^{1}$ Department of Electronic Engineering, Pukyong National University, Nam-gu, Busan 608-737, Korea

${ }^{2}$ Eyemax Co., Ltd., Korea

${ }^{+}$Corresponding author: thkwon@pknu.ac.kr

(Received: Dec. 18, 2013, Revised: Jan. 8, 2014, Accepted: Jan. 9, 2014)

This is an Open Access article distributed under the terms of the Creative Commons Attribution Non-Commercial License(http://creativecommons.org/ licenses/bync/3.0) which permits unrestricted non-commercial use, distribution, and reproduction in any medium, provided the original work is properly cited. enables long-term healthcare monitoring and can obtain electrocardiogram signal without obstructing, contacting, and disturbing the user. Such monitoring system is important for early detection of a heart abnormality in a person. The capacitivecoupled ECG was first introduced by Lopez and Richardson [1]. Then, some researchers expanded it into various environments. Lim et al. [2] and Ueno et al. developed a heart monitoring system in office chair and mattress environment using flexible fabric electrode [3]. Leonhardt el al. [4] applied their idea on insulated electrode implemented in a car-environment system. Lee et al. [5] proposed thin and flexible electrodes for wearable ECG system to build a system that can measure ECG in remote areas. These examples prove that this capacitive technique is quite popular to obtain ECG signal in daily life without any disturbance [6].

However, the current problem is to separate a clean ECG signal from the noise. A small ECG signal of approximately $0.5-4 \mathrm{mV}$ is always mixed with noise from the environment around the measurement system, which is a baseline noise due to respiratory activity, Electromyogram (EMG) noise due to electrical activity of the skeletal muscles, and motion artifact noise due to the contact between the body and electrodes. Various filtering techniques have been proposed by some researchers, such as the adaptive filter combined with an accelerometer by Raya et al. [7], signal averaging and filtering algorithm by Gautam et al. [8], and another method that used wavelet to remove artifact noise by Lee [9]. In 
this paper, we propose a filter that is a combination of an analog filter and a digital extended Kalman filter (EKF).

EKF is a nonlinear version of the Kalman filter, which is chosen because ECG measurement is a nonlinear dynamic system [10]. The implementation of EKF in filtering ECG signal is quite interesting. The researcher in [11] tried to filter the ECG signals offline obtained from the physioNet ECG database using EKF. Niknazar el al. [12] proposed an EKF-based filter to extract electrocardiograms from a single channel, as encountered in fetal ECG extraction from abdominal sensors. Thus, we try to embed the EKF into a microcontroller of an ECG sensor interface to reduce the noise from capacitive-coupled sensors in real time.

This paper was motivated by the following aims: 1) to implement EKF, which is already proven to be a good filter for ECG filtering, into a noncontact ECG measurement sensor interface for real-time measurement to obtain clear and stable ECG signals; 2) to build an interface that can process innovative signal acquisition from a capacitive-coupled sensor combined with an EKF filter; and 3) to implement real-time processing in android mobile devices. The processing algorithm is an expansion of [13], which can process the ECG and locate the QRS wave in an ECG signal. The located QRS wave is then used by the application to determine the heart rate of the user. Information on the heartbeat rate is very important in particular ECG measurement because it is used for further heart analysis such as heart-rate variability analysis and heart abnormality detection.

\section{SYSTEM DESIGN}

\subsection{Capacitive-coupled noncontact ECG}

The developed system applies indirect contact to the patient body. It enables long-term health-care monitoring without disturbing the daily life of a patient. The system consists of two capacitive electrodes, an analog signal processor, a microcontroller, and an interface. The capacitive electrodes are connected to an electronic circuit, which has a high-input impedance amplifier, an instrumentation amplifier, a notch filter, and a band-pass filter for analog signal processing. The block diagram of the overall system is shown in Fig. 1.

A driven-right-leg (DRL) grounding circuit is added to suppress the power-line interference, which is called a common-mode noise. A sheet of conductive textile acts as the ground plane. The

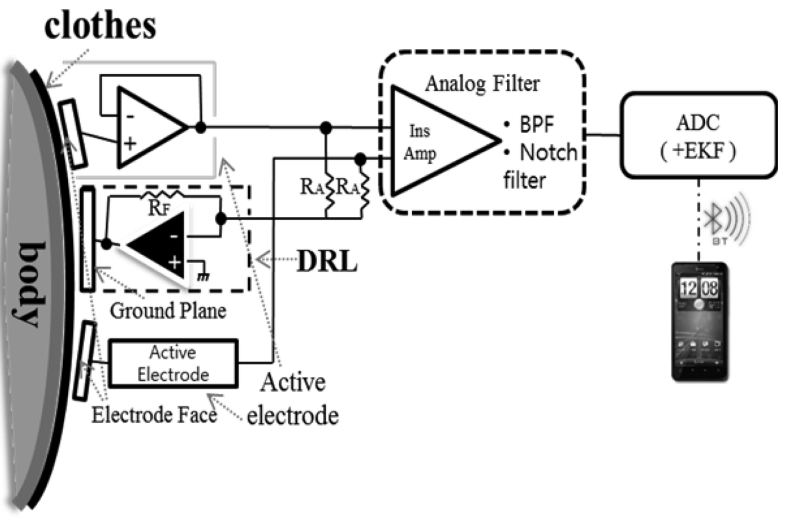

Fig. 1. Block diagram of the capacitive-coupled ECG system.

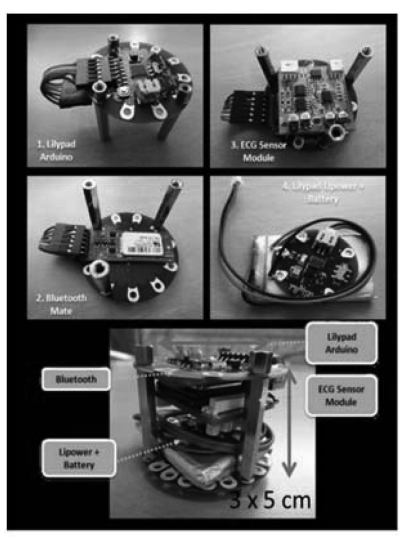

$<$ System Design >

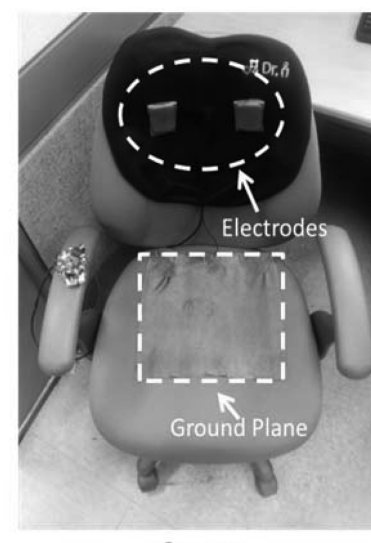

$<$ Sensor >
Fig. 2. Sensors and system design.

ground-plane electrode connected to the instrument ground is driven by the inverse of the common-mode noise component. The sum of the electrode output signal is fed back via an inverting amplifier in the circuit. Resistors $\mathrm{R}_{\mathrm{A}}$ and $\mathrm{R}_{\mathrm{F}}$ adjust the DRL gain, which is chosen to be 1000 in this particular system.

Furthermore, the analog-to-digital converter (ADC) and EKF step is performed by a microcontroller. In this application, the capacitive-coupled system is integrated into an office chair. Fig. 2 shows the electrodes at the backrest and a sheet of conductive textile laid on the seat of the chair as a ground plane.

The methodology of filtering the noisy ECG signal is shown in Fig. 3. The noisy ECG signal measured by the capacitivecoupled electrodes is processed by the analog signal-processing part using the band-pass filter from 0.04 to $100 \mathrm{~Hz}$. This signal directly goes to the microcontroller for conversion into a digital signal and is filtered by the EKF. The digital signal from the sensors is compared with the synthetic ECG signal in the EKF. Then, an innovative signal is obtained, which is called as the EKF-ECG. 


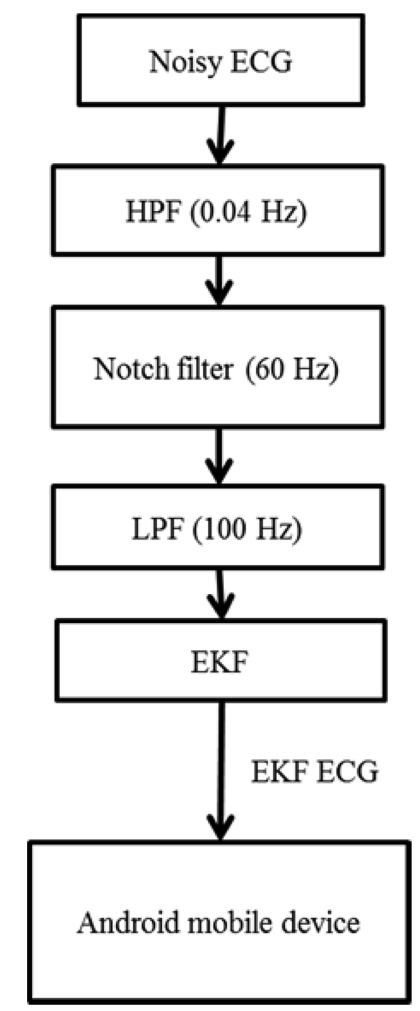

Fig. 3. Block diagram of the filtering process.

\subsection{EKF}

EKF is another version of the Kalman filter, which is specifically developed for nonlinear dynamic models. We assume that a discrete-time nonlinear system is represented as follows:

$$
\begin{aligned}
& x_{k+1}=f\left(x_{k}, w_{k}, k\right) \\
& y_{k}=g\left(x_{k}, v_{k}, k\right) .
\end{aligned}
$$

The nonlinear model in equation (1) can be approximated linearly near a desired reference point $\left(\hat{x}_{k}, \hat{w}_{k}, \hat{v}_{k}\right)$, which leads to the following linear approximation model:

$$
\begin{aligned}
& x_{k+1} \approx f\left(\hat{x}_{k}, \hat{w}_{k}, k\right)+A_{k}\left(x_{k}-\hat{x}_{k}\right)+F_{k}\left(w_{k}-\hat{w}_{k}\right) \\
& y_{k} \approx g\left(\hat{x}_{k}, \hat{v}_{k}, k\right)+C_{k}\left(x_{k}-\hat{x}_{k}\right)+G_{k}\left(v_{k}-\hat{v}_{k}\right),
\end{aligned}
$$

where

$$
\begin{aligned}
& A_{k}=\frac{\partial F}{\partial x}, x=\hat{x}_{k} ; \quad C_{k}=\frac{\partial G}{\partial x}, x=\hat{x}_{k} \\
& F_{k}=\frac{\partial F}{\partial w}, w=\hat{w}_{k} ; G_{k}=\frac{\partial G}{\partial v}, v=\hat{v}_{k}
\end{aligned}
$$

$x_{k}$ is the state vector, $w_{k}$ and $v_{k}$ are the process and measurement noise vectors with associated covariance matrices $Q_{k}=E\left\{w_{k} w_{k}^{T}\right\}$ and $R_{k}=E\left\{v_{k} v_{k}^{T}\right\}$. Then, the extended Kalman equation can be expressed by equations (7)-(12).

$$
\begin{aligned}
& \hat{x}_{k+1}^{-}=\mathrm{f}\left(\hat{x}_{k}^{+}, \mathrm{w}, \mathrm{k}\right) ; \mathrm{w}=\bar{w}_{k} \\
& P_{k+1}^{-}=A_{k} P_{k}^{+} A_{k}^{T}+Q_{k} \\
& r_{k}=y_{k}-q\left(\hat{x_{k}}, v, k\right), v=\bar{v}_{k} \\
& K_{k}=P_{k}^{-} C_{k}^{T}\left[C_{k} P_{k}^{-} C_{k}^{T}+R_{k}\right]^{-1} \\
& \hat{x}_{k}^{+}=\hat{x}_{k}^{-}+K_{k} r_{k} \\
& P_{k}^{+}=P_{k}^{-}-K_{k} C_{k} P_{k}^{-},
\end{aligned}
$$

where $r_{k}$ is defined as the innovative signal, $x_{k}^{+}$and $P_{k}$ are the a posteriori estimations of the state vector and state covariance matrices at time instant $k$, respectively, $\bar{x}_{k}^{-}$and $P_{k}^{-}$are the $a$ priori estimations of the state vector and state covariance matrix at time instant $k$, respectively, and $K_{k}$ is the Kalman filter gain.

\subsection{Synthetic electrocardiogram}

The dynamic model of synthetic electrocardiogram signals was proposed by McSharry et al. [10]. This model is based on the Cartesian coordinates and has various numbers of parameters related to one heartbeat of a human, which makes it capable of generating realistic synthetic electrocardiogram signal. A normal cycle of the ECG corresponds to one heartbeat, which is generally represented by the letters $\mathrm{P}, \mathrm{Q}, \mathrm{R}, \mathrm{S}$, and T. The dynamic model consists of a set of nonlinear dynamic state equation in the Cartesian coordinates.

$$
\begin{aligned}
& \dot{x}=p x-\omega y \\
& y=p y+\omega x \\
& \dot{z}=-\sum_{i \in\{P, Q, R, S, T\}} \alpha_{i} \cdot \Delta \theta_{i} \exp \left(-\frac{\Delta \theta_{i}^{2}}{2 b_{i}^{2}}\right)-\left(z-z_{0}\right),
\end{aligned}
$$

where $x, y$ and $z$ are the state variables that reflect the quasiperiodicity of the ECG in the trajectory $(x, y)$ plane and inter-beat variation in the ECG in the $z$ direction. The parameter values of a typical ECG model are listed in Table 1.

Sameni et al. [9] transformed the model into a polar form. The polar form of the model is expressed as

Table 1. Parameters of the ECG model

\begin{tabular}{cccccc}
\hline \hline Index $(i)$ & $\mathrm{P}$ & $\mathrm{Q}$ & $\mathrm{R}$ & $\mathrm{S}$ & $\mathrm{T}$ \\
\hline Time $(\mathrm{s})$ & -0.2 & -0.05 & 0 & 0.05 & 0.3 \\
$\theta_{\mathrm{i}}(\mathrm{rad})$ & $-\pi / 3$ & $-\pi / 12$ & 0 & $\pi / 12$ & $\pi / 2$ \\
$A_{\mathrm{i}}$ & 20 & -50 & 300 & -75 & 75 \\
$B_{\mathrm{i}}$ & 0.25 & 0.1 & 0.1 & 0.1 & 0.4 \\
\hline
\end{tabular}




$$
\begin{aligned}
& \dot{r}=r(1-r) \\
& \dot{\theta}=\omega \\
& \dot{z}=-\sum_{i \in\{P, Q, R, S, T\}} \alpha_{i} \cdot \Delta \theta_{i} \exp \left(-\frac{\Delta \theta_{i}^{2}}{2 b_{i}^{2}}\right)-\left(z-z_{0}\right)
\end{aligned}
$$

The simplified model of equations (16)-(18) consists of the state equation and observation equation [2]. The state equation describes the ECG dynamics such as the PQRST morphology.

$$
\begin{aligned}
& \theta_{k+1}=\left(\theta_{k}+\omega \Delta\right) \\
& z_{k+1}=-\sum_{i \in\{P, Q, R, S, T\}} \Delta \cdot \alpha_{i} \cdot \Delta \theta_{i} \exp \left(\frac{\Delta \theta_{i}^{2}}{2 b_{i}^{2}}\right)+z_{k}+N . \Delta .
\end{aligned}
$$

$z_{k}$ is a discrete synthetic ECG sample at time $k$, and $\theta_{\mathrm{k}}$ is the angular position. $\alpha_{i}, b_{i}$ and $\theta_{i}$ represent the amplitude, angular width, and position of $i$, respectively, which correspond to the PQRST sub-waveform. $\Delta$ is the sampling time, $N$ is a random additive noise, and $\Delta \theta_{i}=\left(\theta-\theta_{i}\right) \bmod (2 \pi)$.

\subsection{Performance evaluation}

Two of the many methods used to measure or evaluate the performance of a filter are the use of the signal-to-noise ratio (SNR) and the mean squared error (MSE). SNR is a measure used to compare the level of a desired signal to that of the background noise, and MSE corresponds to the expected value of the squarederror loss and measures the average of the squares of the error. The equations for calculating the SNR and MSE are presented in equations (21)-(23), where $x(t)$ is the ECG signal, $n(t)$ is the added noise to the signal, and $\hat{x}(t)$ is the filtered signal.

$$
\begin{aligned}
& S N R_{I N}[d B]=10 \log _{10}\left(\frac{\sum_{i}|x(i)|^{2}}{\sum_{i}|n(i)|^{2}}\right) \\
& S N R_{\text {OUT }}[d B]=10 \log _{10}\left(\frac{\sum_{i}|x(i)|^{2}}{\sum_{i}|x(i)-\hat{x}(i)|^{2}}\right) \\
& \operatorname{MSE}=\frac{\sum_{i}|x(i)-\hat{x}(i)|^{2}}{N}
\end{aligned}
$$

\subsection{QRS detection and heart-rate calculation}

In our system, we implement algorithm to process the ECG data from the sensor and to detect the heartbeat from a particular innovative ECG signal. The processing steps of the algorithm [12] are as follows: (i) band-pass filtering composed of cascaded high-

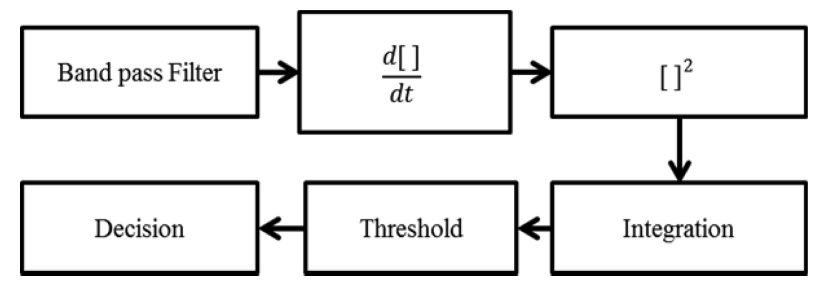

Fig. 4. Processing and heart-rate algorithm.

and low-pass filters; (ii) differentiation, which is a standard technique for finding the high slopes (distinguishing the QRS complex from the other ECG waves); (iii) squaring, to make all data positive; (iv) moving-window integration (in our case with a sampling rate of $200 \mathrm{~Hz}$, the size of the window is 30 samples); (v) thresholding, to identify the peaks of the signal; and (vi) deciding, to detect the QRS complex. A peak must be recognized as QRS in the integrated and filtered waveforms.

\section{EXPERIMENTAL RESULTS}

\subsection{EKF performance analysis}

To investigate the EKF performance of the proposed ECG measurement system, we test our system using Matlab, as shown in Fig. 5. The ECG signal is recorded from a healthy 22-year-old male subject without body movement during the measurement.

Fig. 5(a) shows the corrupted ECG signal with an input SNR of $39.1 \mathrm{~dB}$. After the filtering, the corrupted noisy ECG signal transformed into the signal shown Fig. 5(b), which clearly shows that the innovative signal by the EKF has lesser noise than the original signal. The EKF suppresses the noise from the signal.

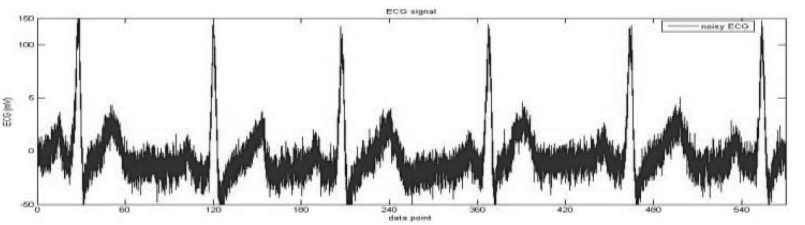

(a)

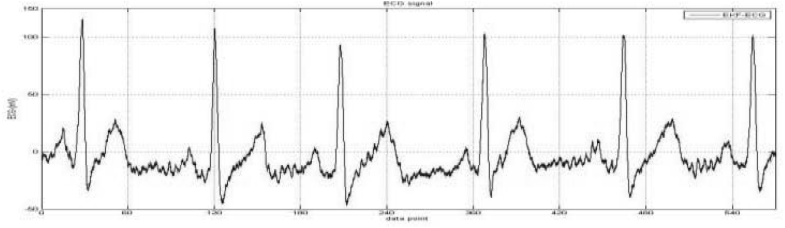

(b)

Fig. 5. ECG results: (a) Noisy recorded ECG signal and (b) EKFECG. 
Table 2. Performance results of the EKF

\begin{tabular}{cccc}
\hline \hline \multicolumn{2}{c}{ Noisy ECG } & \multicolumn{2}{c}{ EKF ECG } \\
\hline SNR [dB] & MSE & SNR [dB] & MSE \\
39.1 & $1.3 \times 10^{-3}$ & 47.79 & $5.4 \times 10^{-4}$ \\
\hline
\end{tabular}

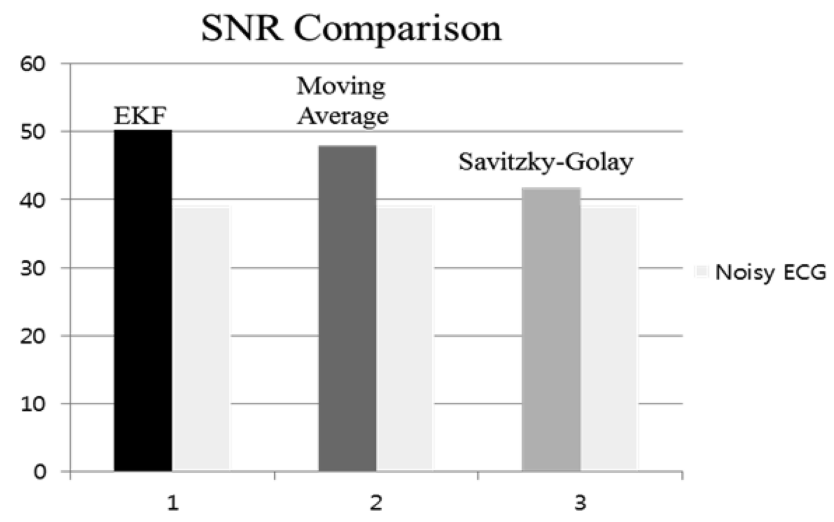

Fig. 6. SNR comparison of the EKF with the other methods that filter ECG signals.

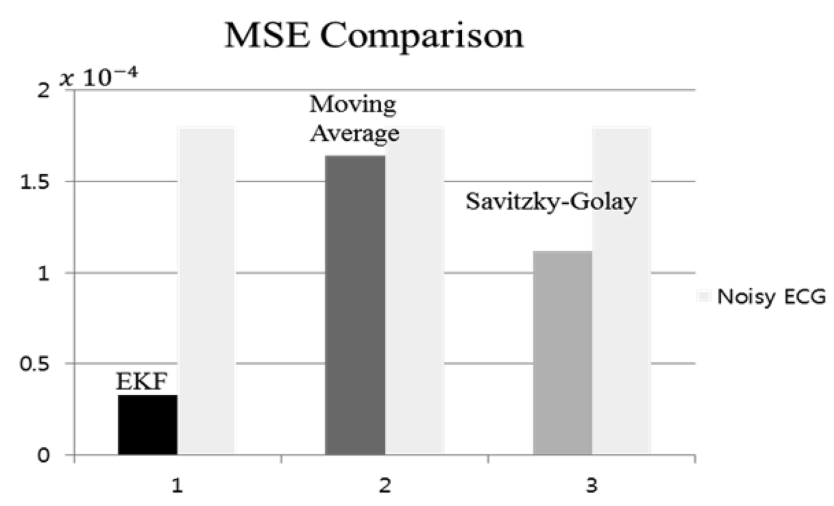

Fig. 7. MSE comparison of the EKF with the other methods that filter ECG signals.
The input and output SNRs and the MSE are defined in equations (21)-(23). Table 2 shows that in the first parameter, which is the SNR, the EKF-ECG signal has a larger value than the noisy signal, which means that the noise has been eliminated by the filter because the higher the SNR is, the better is the system signal quality. In the second parameter, which is the MSE, the noisy ECG reaches $1.3 \times 10^{-3}$. On the other hand, the EKF-ECG suppresses it into $5.4 \times 10^{-4}$, which means that the EKF-ECG signal has lesser error than the noisy one in terms of this parameter.

We also tested other filtering methods that reduce the noise from the same recorded ECG signal. The moving average and Savitzky-Golay methods are well-known methods of reducing noise in an ECG signal. We compare our proposed EKF with the other two filter methods. Figs. 6 and Fig. 7 show that the EKF is more powerful than the other two methods. It has the lowest MSE and the highest SNR among all the above mentioned methods.

\subsection{Real-time embedded EKF}

An ECG data acquisition device is developed on the Lilypad Arduino with an ATMega328 as a microcontroller and ADC. The system uses a $200 \mathrm{~Hz}$ sampling frequency. Electrodes, which are attached to the back of the chair, have an area of 30 $\mathrm{cm}^{2}$ and are separated by approximately 10 in horizontal. The data from the ADC output are filtered by the EKF algorithm embedded in the Arduino and plotted in Fig. 9, which shows the ECG signal obtained using our measurement system after the EKF filtering.

Some experiments are performed to check the robustness of the proposed system. The first experiment checks the system

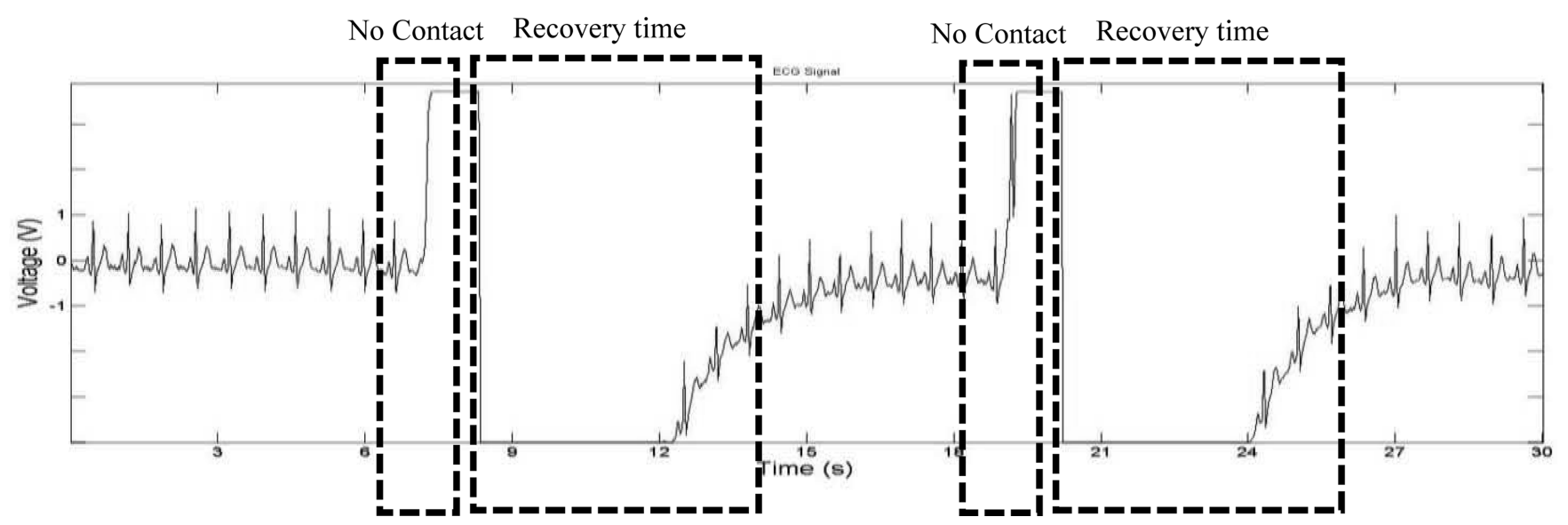

Fig. 8. System performance with noise from body movement. 


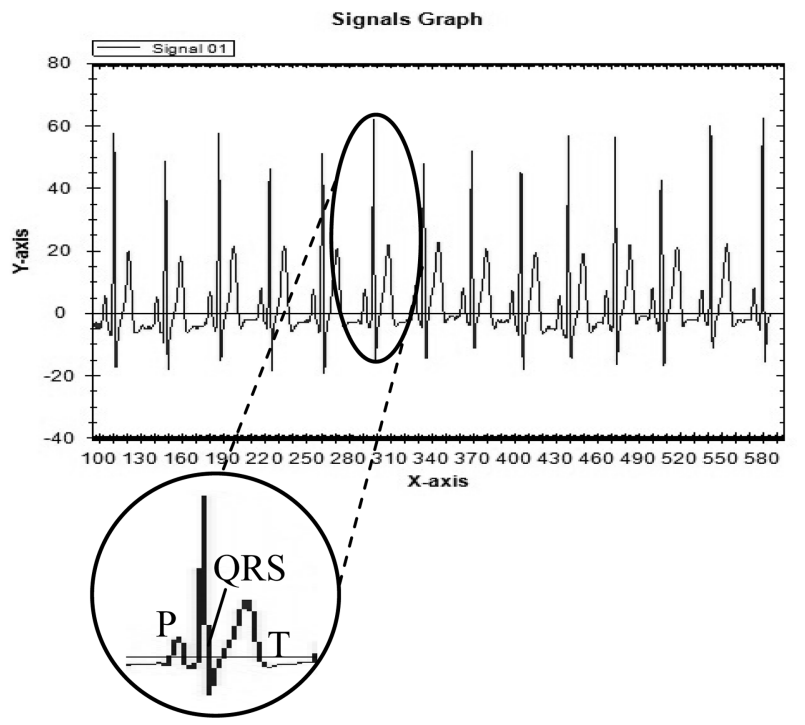

Fig. 9. Clear ECG signal in real time using the embedded EKF.

performance against body movement. Fig. 8 shows that the system needs some time to recover the ECG signal when a change in the contact area with the body occurs. The recovery time is related to the time constant of the High Pass (HP) filter, i.e., it is related to humidity. In most cases, high humidity reduces the resistance of the coupling and clothing. The discharge time of the coupling capacitance and the recovery time are also reduced.

Another experiment that uses different clothes thicknesses is performed, as shown in Fig. 10. The ECG signal obtained from thicker clothes has more noise than those of the thinner ones, especially in the PT wave of the ECG signal. Although this particular experiment shows the effect on the obtained ECG, the system still shows a clear QRS wave, which is the most important information in an ECG signal.

\subsection{Heart-rate detection}

To achieve a ubiquitous monitoring, we try to implement the signal processing and monitoring part in an android mobile device in this study. The idea of implementing the signal-processing part in android mobile devices is our way of distinguishing our system from the previous ones because most previous systems uses only the $\mathrm{PC}$ as an interface, which do not have the advantage of being a ubiquitous health-care system.

The processing algorithm is implemented in android devices for real-time peak detection of the heart activity, which is important information for detailed health-care analysis. The processing algorithm steps from the original signal into the peak QRS

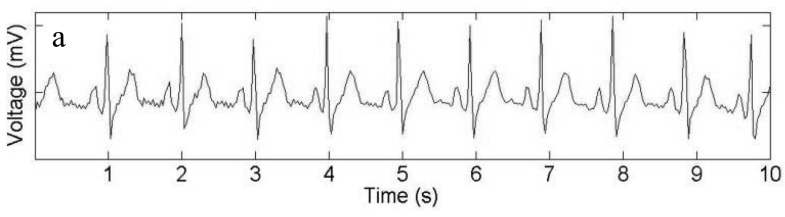

(a)

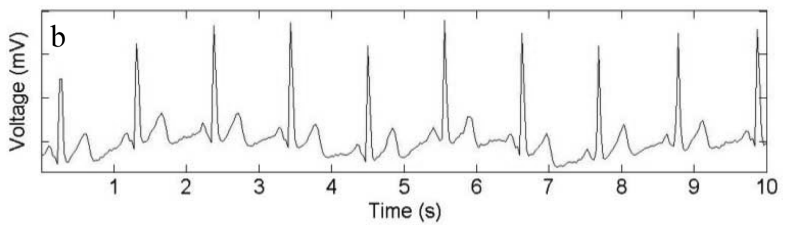

(b)

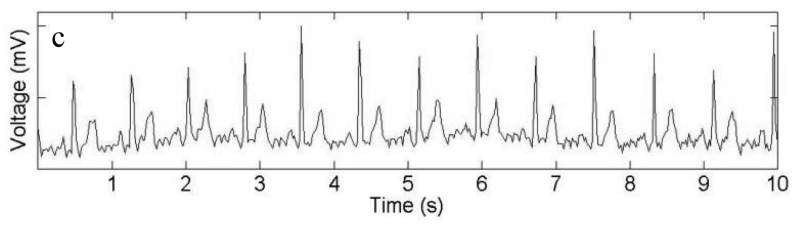

(c)

Fig. 10. Comparison of the ECG signal with different cotton wool shirt thicknesses; (a) $0.5 \mathrm{~mm}$, (b) $1 \mathrm{~mm}$, and (c) 3 $\mathrm{mm}$.

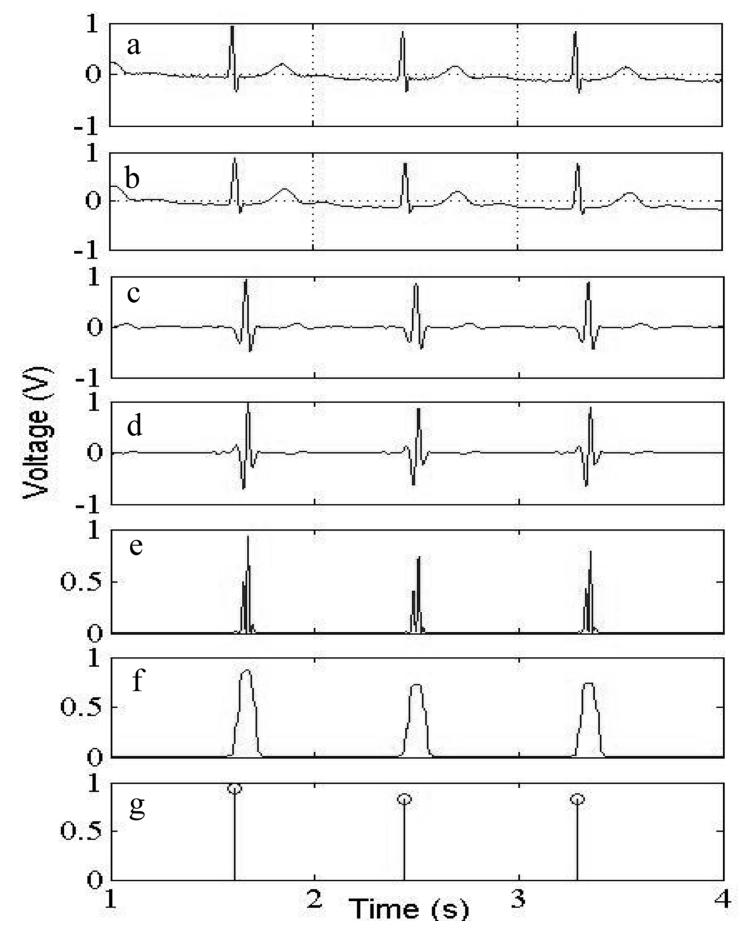

Fig. 11. Processing algorithm; (a) Original signal, (b) low-pass filter, (c) high-pass filter, (d) differentiation, (e) squaring, (f) integration, and $(\mathrm{g})$ peak detection.

detection are shown in Fig. 11.

Fig. 11(g) shows the detected peak as symbolized by the impulse signal. One impulse represents one heartbeat. By 


\section{ORs Bluetooth}

\section{Turn On or Off}
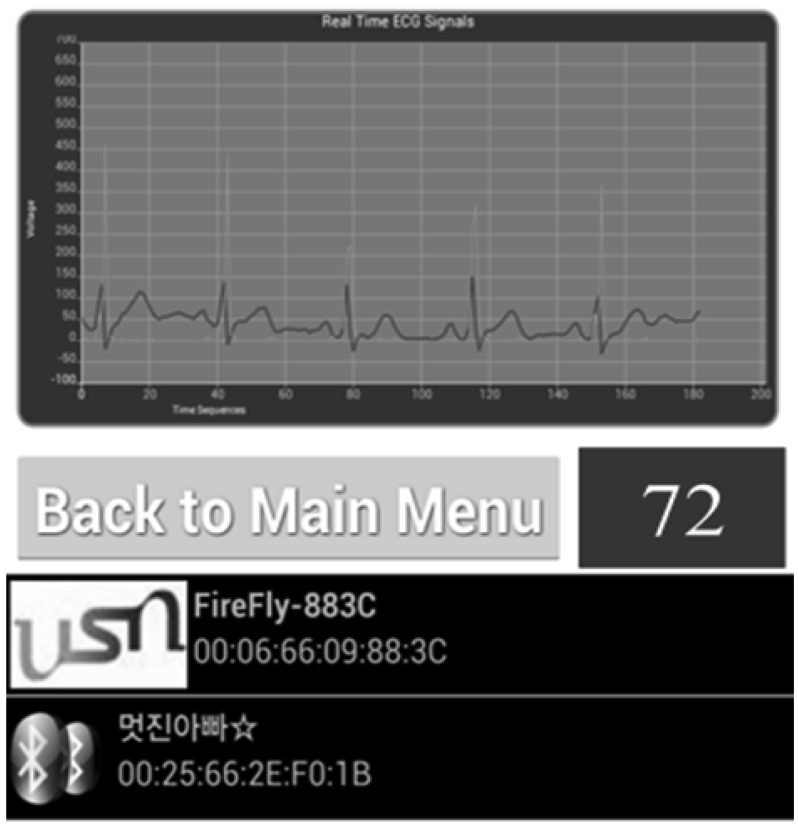

Fig. 12. Android application of the ECG monitoring system.

measuring the consecutive intervals between the heartbeats, the heart rate can be easily calculated. The heart rate unit is expressed in beats per minute (bpm).

$$
\mathrm{HR}(\mathrm{bpm})=\frac{60}{R R \operatorname{Intervals}(s)}
$$

Fig. 12 shows the application during peak detection, as shown by the green line, and the measured heart rate sample is $72 \mathrm{bpm}$. The red line represents the real ECG signal read from our noncontact ECG measurement system.

\section{CONCLUSIONS}

In this paper, noncontact ECG monitoring using capacitivecoupled electrode has been investigated for nonintrusive ECG measurement in daily life. The EKF was proposed for the filtering part to obtain clear ECG signal. The aim of this filtering system is to obtain a stable ECG signal and to improve the SNR by combining the common analog signal processing and digital EKF filtering.

The EKF was designed and implemented for the denoising capacitive-coupled ECG measurement system. The proposed method eliminated the noise from the signal. It increased the SNR of the noisy ECG signal to $47.79 \mathrm{~dB}$, which means that the noise was eliminated by the filter because the higher the SNR is, the better is the signal quality of the system.

To achieve a ubiquitous monitoring system, this paper has proposed an android-based application in mobile devices as an interface. The particular application can process the innovative ECG signal from the sensor and the filter to be used later in detecting the heartbeat peak and calculating the heart rate of the user.

\section{ACKNOWLEDGMENT}

This research was supported by the Ministry of Education, Science, and Technology (MEST) and the National Research Foundation of Korea (NRF) through the Human Resource Training Project for Regional Innovation.

\section{REFERENCES}

[1] A. J. Lopez and P. C. Richardson, "Capacitive electrocardiographic and bioelectric electrodes", IEEE Trans. Biomed. Eng., vol. 16, pp. 99, Jan. 1969.

[2] Y. G. Lim, K. K. Kim, and K. S. Park, "ECG measurement on a chair without conductive contact", IEEE Trans. Biomed. Eng., vol. 53, pp. 956-959, May 2006.

[3] A. Ueno, Y. Akabane, T. Kato, H. Hoshino, S. Kataoka, Y. Ishiyama "Capacitive sensing of electrocardiographic potential through cloth from the dorsal surface of the body in a supine position: A preliminary study", IEEE Trans. Biomed. Eng., vol. 54, no. 4, pp. 759-766, 2007.

[4] S. Leonhardt and A. Aleksandrowicz, "Non-contact ECG monitoring for automotive application", in Proc 5th Int. BSN Conf., pp.183-185, Hong Kong, China, 2008.

[5] S. M. Lee, K. S. Sim, K. K. Kim, Y. G. Lim, and K. S. Park, "Thin and flexible active electrodes with shield for capacitive electrocardiogram measurement", Med Biol. Eng. Comput., vol. 48, pp. 447-457, 2010.

[6] J. H. Kim, S. C. Lee, B. G. Lee, and W. Y. Chung, "Mobile healthcare system based on bluetooth medical device", $J$. Sensor Sci. \& Tech., vol. 21, no. 4, pp. 241-248, 2012.

[7] M. A. D. Raya and L. G. Sison, "Adaptive noise cancellation of motion artifact in stress ECG signals using accelerometer", Proc. $2^{\text {nd }}$ Joint EMBS/BMES Conference. 2002.

[8] A. Gautam, Y. D. Lee, and W. Y. Chung, "ECG signal denoising with signal averaging and filtering algorithm", Third International Conference on Convergence and Hybrid Information Technology, 2008.

[9] S. M. Lee, K. K. Kim, and K. S. Park, "Wavelet approach to artifact noise removal from capacitive coupled electrocardiograph", 30th Annual International IEEE EMBS Con- 
ference, Canada, pp. 2944-4947, 2008.

[10] P. E. McSharry, G. D. Clifford, L. Tarassenko, and L. A. Smith, "A dynamical model for generating synthetic electrocardiogram signals", IEEE Transaction on Biomedical Engineering, vol. 50, no. 3, pp. 289-294, 2003.

[11] R. Sameni, M. B. Shamsollahi, C. Jutten, and M. B. Zadeh, "Filtering noisy ECG signals using the extended Kalman filter based on a modified dynamic ECG model", IEEE
Computes in Cardiology, vol. 32, pp. 1017-1020, 2005.

[12] M. Niknazar, B. Rivet, and C. Jutten, "Fetal ECG extraction by extended state Kalman filtering based on single-channel recordings", IEEE Transactions on Biomedical Engineering, vol. 60, no. 5, pp 1345-1352, 2013.

[13] J. Pan and W. J. Tompkins "A real-time QRS detection algorithm", IEEE Trans Biomed. Eng, vol. 32, no. 3, pp. 230-236, 1985. 